\title{
Macro- and micro-scale studies on U(VI) immobilization in hardened cement paste
}

\author{
E. Wieland • N. Macé • R. Dähn • \\ D. Kunz $\cdot$ J. Tits
}

Received: 20 July 2010/Published online: 5 September 2010

(C) Akadémiai Kiadó, Budapest, Hungary 2010

\begin{abstract}
Wet chemistry and synchrotron-based (micro-) spectroscopic investigations have been carried out to determine the uptake and speciation of $\mathrm{U}(\mathrm{VI})$ in hardened cement paste (HCP). The wet chemistry experiments included kinetic studies and the determination of the sorption isotherm. The latter measurements allowed conditions for linear sorption to be distinguished from those where precipitation occurred. Micro-X-ray fluorescence and X-ray absorption spectroscopy ( $\mu$-XRF/XAS) were used to determine the elemental distribution and the coordination environment of $\mathrm{U}(\mathrm{VI})$ in an intact $\mathrm{HCP}$ sample at the atomic level. The sample was prepared by indiffusion of $\mathrm{U}(\mathrm{VI})$ into HCP over 9 months. Micro-XRF maps revealed a heterogeneous distribution of $U(V I)$ in a ten micron thick layer on the surface of the HCP disk. Micro-XAS measurements on a U(VI) hot spot showed that the coordination environment of $\mathrm{U}(\mathrm{VI})$ is similar to that in $\mathrm{U}(\mathrm{VI})$ doped HCP and in C-S-H sorption samples. To the best of our knowledge this is the first synchrotron-based micro-spectroscopic study on the speciation of diffusing uranyl ions with micro-scale spatial resolution.
\end{abstract}

Keywords Uranium · Cement $\cdot$ Micro-spectroscopy · Diffusion

E. Wieland $(\bowtie) \cdot$ N. Macé $\cdot$ R. Dähn $\cdot$ D. Kunz $\cdot$ J. Tits Laboratory for Waste Management, Nuclear Energy and Safety Research Department, Paul Scherrer Institut, 5232 Villigen PSI, Switzerland

e-mail: erich.wieland@psi.ch

Present Address:

N. Macé

CEA Saclay, DEN/DANS/DPC/SECR, 91191 Gif-sur-Yvette

Cedex, France

\section{Introduction}

Cementitious materials are being used worldwide for the stabilization of low- and intermediate-level radioactive waste (L/ILW) and for the construction of the engineered barrier systems [1]. In safety assessments it is considered that the mobility of radionuclides in the cementitious near field is significantly reduced due to interaction with hardened cement paste (HCP). Uranium, an element of the actinide series used in nuclear fuel fabrication, is present in $\mathrm{L} / \mathrm{ILW}$. Uranium(VI) is expected to be the dominant redox state under the relevant repository conditions, i.e., slightly oxidising to reducing redox conditions, and therefore the behaviour of $\mathrm{U}(\mathrm{VI})$ in a cement-based repository is of considerable interest in connection with long-term predictions of the safe disposal of radioactive waste.

While numerous sorption studies with $\mathrm{U}(\mathrm{VI})$ on HCP and cement minerals have been reported (e.g., [2-8]), the sorption mechanism of $\mathrm{U}(\mathrm{VI})$ uptake by HCP is still poorly understood at the atomic level. Extended X-ray absorption fine structure (EXAFS) spectroscopy studies on $\mathrm{U}(\mathrm{VI})$ loaded untreated and hydro-thermally treated cement samples revealed preservation of the uranyl $\left(\mathrm{UO}_{2}{ }^{2+}\right)$ moiety, indicating that the $+\mathrm{VI}$ redox state was stable [9, 10]. The EXAFS studies further showed that U(VI) equilibrated with hydrothermally altered concrete formed oligomeric surface complexes or surface precipitates as indicated from the U-U backscattering contributions observed in the EXAFS spectra. U(VI) interaction with the surfaces of cement minerals was suggested to occur via sharing of equatorial oxygen atoms. EXAFS studies performed on $\mathrm{U}(\mathrm{VI})$ doped calcium silicate hydrates (C-S-H), which is the most important constituent of $\mathrm{HCP}$, suggested a chemical environment of $\mathrm{U}(\mathrm{VI})$ 
similar to that of $\mathrm{U}(\mathrm{VI})$ in the uranyl silicate mineral uranophane [11]. Nevertheless, the coordination environment of U(VI) species immobilized in HCP, and in particular the cement mineral responsible for U(VI) immobilization, remains unknown.

The present study was undertaken with the aim of quantifying U(VI) uptake by $\mathrm{HCP}$ and elucidating the coordination environment of $\mathrm{U}(\mathrm{VI})$ in an intact $\mathrm{HCP}$ sample at the atomic level. The latter sample was prepared by in-diffusion of U(VI) into HCP. Synchrotron-based micro-spectroscopy was used to determine the speciation of U(VI) diffusing into the intact HCP disk. The spectroscopic results were compared with those determined for U(VI) sorbed onto crushed HCP and synthetic C-S-H as well as relevant uranyl-bearing minerals.

\section{Materials and methods}

Throughout this study the solutions were prepared using Fluka or Merck "pro analysis" chemicals and deionised, decarbonated water (Milli-Q water) generated by a water purification system Milli-Q Gradient A10 (Millipore, Billerica, USA). Prior to use, the $40 \mathrm{~mL}$ polyallomere centrifuge tubes (Beckman Instruments Inc., Fullerton, USA) employed for the wet chemistry experiments and sample preparation were washed, left overnight in a solution of $0.1 \mathrm{M} \mathrm{HCl}$, and thoroughly rinsed with de-ionised water. Sample preparation involving alkaline solutions and solids as well as the experimental studies (sorption and diffusion) were performed in a glove box under a $\mathrm{N}_{2}$ atmosphere $\left(\mathrm{O}_{2}, \mathrm{CO}_{2}<2 \mathrm{ppm}\right)$ in order to avoid $\mathrm{CO}_{2}$ contamination.

\section{Materials}

Hardened cement paste samples were prepared using commercial sulphate-resisting Portland cement CEM I $52.2 \mathrm{~N}$ HTS, denoted as HTS cement (HTS = Haute Teneur en Silice, Lafarge, France) and a procedure described earlier [12, 13]. The chemical composition of cement was reported elsewhere [14]. The cement pastes were prepared using a water-to-cement ratio of 1.3 and hydrated for more than 8 years. The crushed HCP material used in the sorption experiments was prepared by grinding intact HCP specimens in a mortar under $\mathrm{CO}_{2}$-free conditions, followed by air-drying in the glove box (relative humidity $\cong 10 \%$ ) and sieving the crushed material to collect the size fraction $\leq 70 \mu \mathrm{m}$. The average surface area was determined to be $57 \pm 4 \mathrm{~m}^{2} \mathrm{~g}^{-1}$ based on $\mathrm{N}_{2}$ sorption BET measurements on four replicates using a Micrometrics Gemini 2360 analyzer. The diffusion sample and the synthetic $\mathrm{C}-\mathrm{S}-\mathrm{H}$ phase were prepared according to procedures reported earlier $[13,15]$.

Sorption and diffusion experiments

Kinetic experiments with U(VI) on HCP were carried out using ${ }^{233} \mathrm{U}$ tracer $\left(t_{1 / 2}=1.59 \times 10^{5}\right.$ years $)$. For the sample preparation $800 \mu \mathrm{L}$ aliquots of a HCP stock suspension (50 $\mathrm{g} \mathrm{L}^{-1}$ ) were mixed with $39.2 \mathrm{~mL}$ artificial cement pore water (ACW; $\mathrm{pH}=13.3$ ) [16] in centrifuge vials (solid-toliquid ratio $\left.(\mathrm{s} / \mathrm{l})=10^{-3} \mathrm{~kg} \mathrm{~L}^{-1}\right)$. The samples were labelled with $1 \mathrm{~mL}{ }^{233} \mathrm{U}(\mathrm{VI})$ solution $(0.2 \mathrm{~mL}$ stock solution $\left(4.33 \times 10^{-3} \mathrm{M}\right)$ diluted in $\left.40 \mathrm{~mL} 0.1 \mathrm{M} \mathrm{HNO}_{3}\right)$ and shaken end-over-end up to 60 days. After centrifugation (60 min at $95,000 \mathrm{~g}$ ) $5 \mathrm{~mL}$ aliquots were withdrawn from the supernatant solutions and mixed with $15 \mathrm{~mL}$ scintillator (Ultima Gold XR, PerkinElmer, Waltham, USA) prior to counting. Samples, standards and blank samples were analysed by radioassay using a Canberra Packard Tri-carb 2250 CA liquid scintillation counter (energy window between 100 and $200 \mathrm{keV}$ ). Standards were prepared by mixing $0.1 \mathrm{~mL}{ }^{233} \mathrm{U}$ tracer solution with $5 \mathrm{~mL} \mathrm{ACW}$ and $15 \mathrm{~mL}$ scintillator, while blank samples contained $5 \mathrm{~mL}$ ACW and $15 \mathrm{~mL}$ scintillator.

The sorption isotherm was determined using a ${ }^{233} \mathrm{U}$ tracer solution and a solution prepared from natural uranium. The HCP suspensions were prepared as described above. For the lower U(VI) concentration appropriate amounts of the ${ }^{233} \mathrm{U}$ tracer solution $\left(2.17 \times 10^{-6} \mathrm{M}\right.$ in $0.001 \mathrm{M} \mathrm{HNO}_{3}$ ) were added while the U(VI) concentrations in the upper range were adjusted by adding appropriate amounts of a $10^{-2} \mathrm{M}$ natural uranium stock solution (uranium-nitrate salt dissolved in $0.001 \mathrm{M} \mathrm{HNO}_{3}$ ). After equilibration for 30 days end-over-end, solid and liquid phases were separated by centrifugation, $5 \mathrm{~mL}$ aliquots withdrawn from the supernatant solutions and analysed by liquid scintillation $\left({ }^{233} \mathrm{U}\right)$ or inductively coupled plasmaoptical emission spectroscopy (ICP-OES) in the case of the samples containing natural uranium.

The portion of uranyl sorbed was determined from the difference of total U(VI) added to the suspensions and the aqueous U(VI) concentration in the supernatant solution. Preliminary test experiments revealed that U(VI) sorption onto the walls of the centrifuge tubes was negligible. Uranyl uptake by HCP was described in terms of a distribution ratio $\left(R_{d}\right)$ as given elsewhere [17].

In-diffusion experiments with U(VI) were carried out using diffusions cells previously developed for out-diffusion experiments as reported elsewhere [13, 18]. A $1 \mathrm{~cm}$ thick HCP disk was glued into the sample holder and mounted between two $100 \mathrm{~mL}$ compartments. The compartments on each side of the HCP disk were filled with ACW. The system was equilibrated for 1 month prior to 
U(VI) addition. An U(VI) precipitate was prepared by mixing an excess of the natural uranium stock solution with ACW and aged for 1 week. The precipitate was separated from solution by centrifugation and introduced into the primary compartment (high concentration side) as an excess U(VI) source for the diffusion experiment. The U(VI) concentration corresponded to the solubility limit of $\mathrm{U}(\mathrm{VI})$ in $\mathrm{ACW}$, which was determined to be $6 \times 10^{-6} \mathrm{M}$ based on the sorption isotherm. The secondary compartment was filled with U(VI) free ACW. After regular time intervals, samples were withdrawn from both compartments and analyzed for $\mathrm{Ca}$ and $\mathrm{U}$. Both concentrations were constant over time in the primary compartment as the added U(VI) precipitate was present in excess while the $\mathrm{Ca}$ concentration in $\mathrm{ACW}$ was constant and corresponded to its initial value $\left([\mathrm{Ca}]_{\mathrm{t}}=1.6 \times 10^{-3} \mathrm{M}\right)$ [16]. Uranium was not detected in the secondary compartment over the course of the experiment ( 9 months), while the $\mathrm{Ca}$ concentration corresponded to that in the primary compartment.

\section{Sample preparation for the spectroscopic investigations}

Ca-uranate $\left(\mathrm{CaUO}_{4} \cdot \mathrm{xH}_{2} \mathrm{O}\right)$ and uranophane were synthesized by Moroni and Glasser [19]. For the EXAFS measurements about $25 \mathrm{mg}$ of each mineral was thoroughly mixed with $250 \mathrm{mg}$ boron nitride (BN, 98\% purity, SigmaAldrich) in a mortar and placed in Plexiglas holders. The holders were sealed with two layers of Kapton tape and, in addition, with thermo-sealed polyethylene bags. The U(VI) doped C-S-H sample (C-S-H/1.8_H $\left.{ }_{2} \mathrm{O} \_309 d\right)$ was prepared according to a procedure described earlier [11]. The synthetic $\mathrm{C}-\mathrm{S}-\mathrm{H}$ phase had a $\mathrm{Ca} / \mathrm{Si}(\mathrm{C} / \mathrm{S})$ ratio of 1.8 . U(VI) stock solution $\left(10^{-2} \mathrm{M}\right)$ was added to $40 \mathrm{~mL} \mathrm{C-S-H}$ suspension to obtain an U(VI) loading of about 2,100 ppm. The suspension was equilibrated end-over-end for 309 days. The HCP sample (HCP_ACW_28d) was prepared by mixing $1 \mathrm{~g}$ crushed $\mathrm{HCP}$ material with $1.1 \mathrm{~mL}$ $\mathrm{U}(\mathrm{VI})$ stock solution $\left(10^{-2} \mathrm{M}\right)$ in $40 \mathrm{~mL}$ ACW. The U(VI) loading was about $2,700 \mathrm{ppm}$, which corresponds to an U(VI) surface loading in the upper range of the linear sorption isotherm. After equilibration for 28 days, solid and liquid phases were separated by centrifugation. The wet pastes were dried in the glove box under a $\mathrm{N}_{2}$ atmosphere for about $24 \mathrm{~h}$, placed into Plexiglas holders and double-sealed as described above.

The diffusion sample was analyzed using micro-X-ray fluorescence ( $\mu$-XRF) and micro-X-ray absorption spectroscopy $(\mu$-XAS). For this, a thin section was prepared from the cross section by polishing the HCP material after pressure impregnation with epoxy resin (Th. Beckmann, Soil morphology, Schwülper-Lagersbüttel, Germany, pers. comm.).
EXAFS, $\mu$-XRF and $\mu$-XAS data collection and reduction

Detailed descriptions of the measurements, data collection and reduction are given elsewhere (Macé, Environ. Sci. Technol., submitted). Briefly, bulk-EXAFS spectra at the U $\mathrm{L}_{\mathrm{III}}$ edge (17166 eV) were collected at BM 20 (ROBL) at the European Synchrotron Radiation Facility (ESRF) in Grenoble, France. Set-up and measuring conditions were selected according to a previous study [11]. The $\mu-\mathrm{XRF} /$ XAS measurements on the diffusion sample were performed at BL 2-3 at the Stanford Synchrotron Radiation Lightsource (SSRL) of the SLAC National Accelerator Laboratory (Menlo Park, CA, USA). The beam line is equipped with a $\mathrm{Si}(111)$ double-crystal monochromator and a Kirkpatrick-Baez (KB) mirror focusing system. The monochromator position was calibrated by assigning the first inflection point of the K-absorption edge of the $\mathrm{Y}$ foil to $17,038 \mathrm{eV}$. The X-ray beam was focussed down to about $2 \times 2 \mu \mathrm{m}^{2}$ spot size. Fluorescence radiation was detected using a single channel VORTEX Si detector. The elemental distribution maps ( $\mathrm{U}, \mathrm{Ca}, \mathrm{K}$ and $\mathrm{Fe}$ ) were recorded using $2 \mu \mathrm{m}^{2}$ pixel size and a dwell time per point of $250 \mathrm{~ms}$. A total of 17 EXAFS spectra were recorded on the selected $\mathrm{U}(\mathrm{VI})$ rich spot to achieve the requested signal-to-noise ratio.

Bulk-EXAFS and $\mu$-EXAFS data reduction and analysis were performed using the SIXPACK and IFEFFIT software packages following standard procedures [20-22]. SIXPACK was applied for dead-time correction and averaging the raw spectra. Reduction and modelling of the EXAFS data were performed with the ATHENA/ARTEMIS software package. After background subtraction, the energy was converted to photoelectron wave vector units $\left(\AA^{-1}\right)$ by assigning the ionization energy of the uranium $\mathrm{L}_{\text {III }}$ edge $(17,166 \mathrm{eV}), E_{0}$, to the first inflection point of the absorption edge. Radial structural functions (RSFs) were obtained by Fourier transforming $k^{3}$-weighted $\chi(k)$ functions between 2 and $16 \AA^{-1}$ for the reference compounds and 2 and $11 \AA^{-1}$ for the U(VI) doped samples. A multi shell approach was employed for data fitting. Theoretical single and multiple scattering paths (SS and MS paths, respectively) were calculated with FEFF8.2 using the $\alpha$-uranophane or soddyite structure as model compounds. Three MS backscattering paths (MS1, MS2 and MS3) were considered in all fits as reported elsewhere [23]. For the fitting some parameters were fixed or linked to reduce the number of free parameters, e.g., the coordination number $(N)$ of the first shell (axial $\mathrm{O}$ atoms) was fixed $(N=2)$ in agreement with the uranyl structure, and the threshold energy $\left(\Delta E_{0}\right)$, which was allowed to vary as a global parameter, was linked for all shells. The fit approach was tested on several reference compounds, i.e., U(VI) nitrate, 
soddyite, becquerelite, $\alpha$-uranophane and $\mathrm{CaUO}_{3.8}$ by comparing fitted data with the crystallographic distances reported in the literature (Macé, Environ. Sci. Technol., submitted).

\section{Results and discussion}

Wet chemistry experiments

The kinetic studies with U(VI) on HCP showed that U(VI) sorption reaches equilibrium after 14 days (Fig. 1a). The $R_{d}$ values determined after 14,32 , and 60 days reaction time agree within the uncertainty range of the measurements (mean value: $R_{d}=2.2 \pm 0.5 \mathrm{~m}^{3} \mathrm{~kg}^{-1}$ ). This value is consistent with those determined earlier under conditions relevant to a "fresh" HCP system, with $R_{d}$ ranging in value between $0.5 \pm 0.1-25 \pm 10 \mathrm{~m}^{3} \mathrm{~kg}^{-1}$ [2-4]. Furthermore, $\mathrm{U}(\mathrm{VI})$ binds more strongly onto HCP than alkaline earth metals, such as $\operatorname{Sr}(\mathrm{II})$ with $R_{d}=0.1 \pm 0.03 \mathrm{~m}^{3} \mathrm{~kg}^{-1}$ [17], under comparable experimental conditions. However, the sorption value is similar to that of bivalent heavy metals, e.g., $\mathrm{Pb}(\mathrm{II})$ where $R_{d}$ values range between 0.1 and $8 \mathrm{~m}^{3} \mathrm{~kg}^{-1}$ $[24,25]$. Note that different sorption mechanisms may be operative at the atomic level $[15,17,24,26]$. A Sr-Ca exchange mechanism on C-S-H was used to model Sr uptake by fresh and degraded HCP $[15,17]$ while either $\mathrm{Ca}-\mathrm{Pb}$ replacement or surface complexation was suggested to control the uptake of $\mathrm{Pb}$ at the molecular scale $[24,26,27]$.

The uptake of U(VI) by HCP at $\mathrm{pH}=13.3$ was found to be linear in the concentration range of dissolved U(VI) below about $6 \times 10^{-6} \mathrm{M}$, suggesting that one type of surface site controls the interaction of U(VI) with HCP (Fig. 1b). Comparison of the $R_{d}$ values determined on HCP with measurements on C-S-H allowed the role of C-S-H in $\mathrm{U}(\mathrm{VI})$ sorption to be assessed. Tits et al. [8] reported sorption values for a $\mathrm{C}-\mathrm{S}-\mathrm{H}$ phase with a $\mathrm{C} / \mathrm{S}$ ratio $=1.1$, which is the highest $\mathrm{C} / \mathrm{S}$ ratio achievable at $\mathrm{pH}=13.3$ without portlandite precipitation, ranging in value between 1 and $10 \mathrm{~m}^{3} \mathrm{~kg}^{-1}$. The $R_{d}$ value for $\mathrm{U}(\mathrm{VI})$ on $\mathrm{HCP}$ was estimated to be about $2.5 \mathrm{~m}^{3} \mathrm{~kg}^{-1}$ based on an average $R_{d}$ value of $5 \mathrm{~m}^{3} \mathrm{~kg}^{-1}$ for $\mathrm{U}(\mathrm{VI})$ on C-S-H and assuming that the portion of C-S-H in HCP is about 50 weight (wt) \% for sulphate-resisting Portland cement CEM I 52.2 N HTS [14]. Thus, the estimated value is in excellent agreement with the experimental data, supporting the idea that C-S-H could be the uptake-controlling cement phase for $\mathrm{U}(\mathrm{VI})$ in HCP.

The formation of a $\mathrm{U}(\mathrm{VI})$ precipitate was indicated in the sorption experiments at higher $\mathrm{U}(\mathrm{VI})$ inventories (Fig. 1b). The solubility limit of $\mathrm{U}(\mathrm{VI})$ at $\mathrm{pH}=13.3$ was found to be about $6 \times 10^{-6} \mathrm{M}$. Tits et al. [8] observed excellent agreement between the above-mentioned,
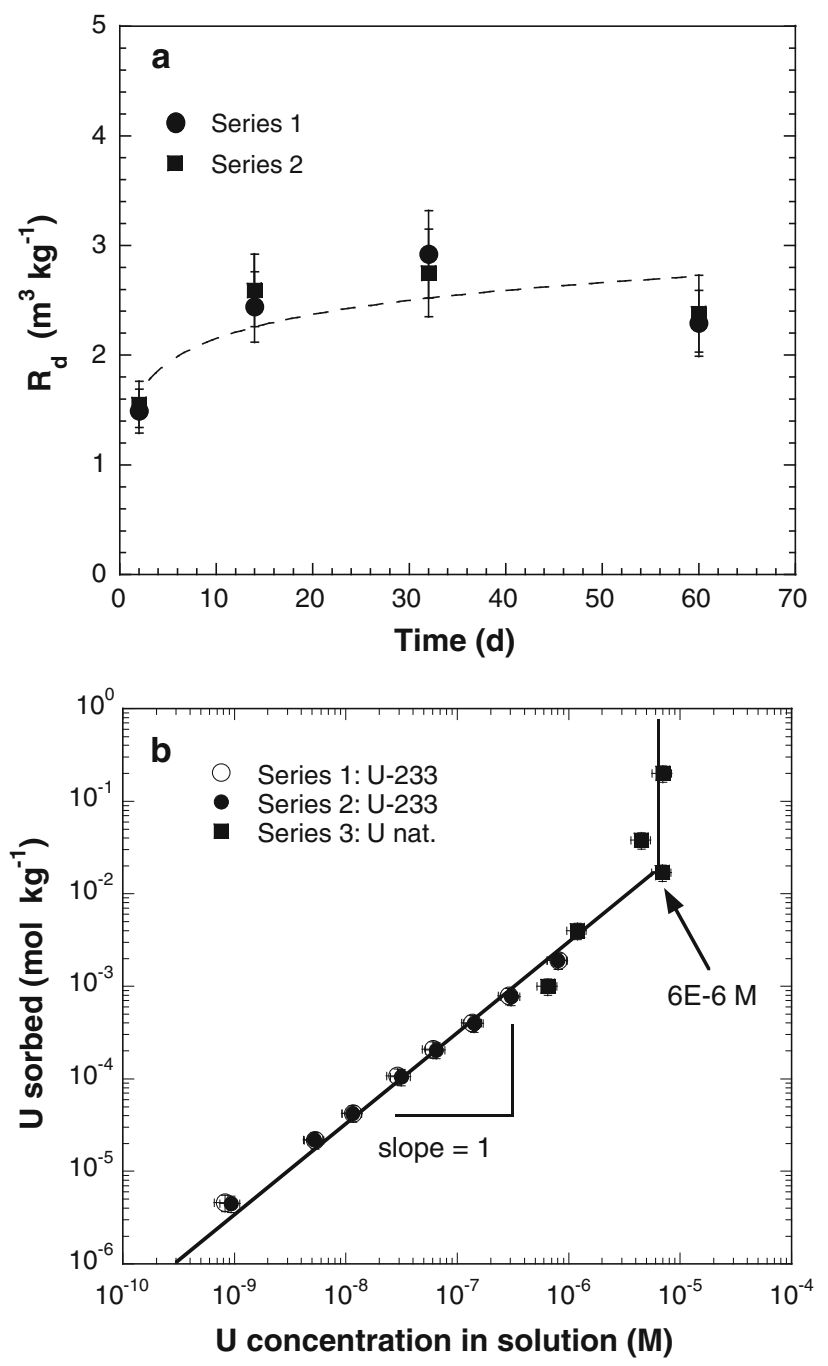

Fig. $1 \mathrm{U}(\mathrm{VI})$ uptake by hardened cement paste at $\mathrm{pH}=13.3$, a time dependence of the uptake process, $\mathbf{b}$ sorption isotherm

experimentally determined $\mathrm{U}(\mathrm{VI})$ concentration in solution at $\mathrm{pH}=13.3$ with predictions made on the assumption that a hydrous Ca-uranate (solubility product $\log _{10} K_{s, 0}^{0}=23.1$ [19]) is the solubility-limiting $\mathrm{U}(\mathrm{VI})$ phase:

$\mathrm{CaUO}_{4}(\mathrm{~s})+4 \mathrm{H}^{+} \Leftrightarrow \mathrm{Ca}^{2+}+\mathrm{UO}_{2}^{2+}+2 \mathrm{H}_{2} \mathrm{O}$

The wet chemistry experiments revealed that C-S-H phases could be the uptake-controlling cement phase in $\mathrm{HCP}$ at low U(VI) inventories while the formation of Ca-uranate might control the $\mathrm{U}(\mathrm{VI})$ concentration in solution at higher U(VI) inventories. Spectroscopic investigations were carried out with the aim of further substantiating these findings and to provide structural information on the U(VI) sorbed species. The results from micro-spectroscopic studies on HCP systems with high U(VI) loadings will be reported elsewhere (Macé, Environ. Sci. Technol., submitted). In this study, selected spectroscopic data on HCP systems with low U(VI) loadings, i.e., with U(VI) surface 
concentrations in the linear range of the sorption isotherm, will be presented and discussed. The set-up used in the $\mathrm{U}(\mathrm{VI})$ in-diffusion experiment is of particular interest as the system was always undersaturated with respect to Ca-uranate formation.

\section{Spectroscopic investigations}

Micro-XRF was used to determine the elemental distribution in the surface layers of both sides of the HCP disk. In Fig. 2 the U, Ca, K, and Fe maps are shown, which were recorded along the surface of the HCP material exposed to the primary compartment. Furthermore, mapping was carried out on the surface of the HCP material exposed to the secondary compartment, revealing that U(VI) was absent in the surface layers (data not shown). This finding confirms that U(VI) did not diffuse through the cement barrier over the course of 9 months. U(VI) was retained within the top surface layer (about $10 \mu \mathrm{m}$ thickness) on the high concentration side.

Figure 2 shows that the distribution of U(VI) in HCP is very heterogeneous. U(VI) seems to be enriched in regions where the $\mathrm{Ca}$ concentration (e.g., spots framed with solid lines and labelled 1-5) is high. These regions indicate the presence of clinker minerals, i.e., alite $\left(\mathrm{C}_{3} \mathrm{~S}\right)$ or belite $\left(\mathrm{C}_{2} \mathrm{~S}\right)$, respectively. An apparent correlation between $\mathrm{K}$ and $\mathrm{U}$ (e.g., spots framed with a broken line and labelled 6 and 7) appears in some regions of the surface layer. At the present time it is uncertain whether the correlation indicates the formation of a K-uranate-type material or is simply due to an artefact caused by an overlap of the $\mathrm{M} \alpha$ and $K \alpha_{1,2}$ emission lines of $U$ and $K$, respectively. Figure 2 also reveals that $\mathrm{Fe}$ and $\mathrm{U}(\mathrm{VI})$ distributions do not correlate, suggesting that Fe-bearing minerals are not important for U(VI) immobilization in HCP.
As stated above, preferential accumulation around clinker minerals is indicated from the XRF maps. During the hydration process, i.e., upon contacting unhydrated cement with water, the clinker minerals (alite, belite, aluminate, and ferrite) start dissolving, thus acting as $\mathrm{Ca}, \mathrm{Si}$, $\mathrm{Al}$, and Fe sources. In combination with the main $\mathrm{S}$ source, gypsum, this results in the formation of the hydrate assemblage with C-S-H as the main constituent [14]. Dissolution of belite is significantly slower than that of alite, while the amount of aluminate and ferrite is very low. Thus, C-S-H phases are expected to form in the highly reactive zones around belite in the long term while alite dissolution gives rise to $\mathrm{C}-\mathrm{S}-\mathrm{H}$ precipitation in the early stages of cement hydration. It should be noted that previous studies already reported the preferential accumulation of $\mathrm{Ni}$ and $\mathrm{Nd}$ in the reactive zones around belite [28, 29]. In the latter studies, $\mathrm{Ni}$ and $\mathrm{Nd}$ containing solutions with relatively high metal concentrations were added to unhydrated cement. Therefore, the metals could accumulate around clinker minerals already in the early stages of cement hydration. In contrast, U(VI) was permitted to diffuse into an almost fully hydrated cement paste in the present study, suggesting that phase transformations due to cement hydration (dissolution of clinker minerals and formation of the hydrate assemblage) should be negligible, and the content of unhydrated clinker minerals should be low. Nevertheless, U(VI) appeared to accumulate preferentially in the chemical reactive zones around clinker minerals, even in a paste that had been aged over a very long time.

Figure 3 shows the bulk- and $\mu$-EXAFS spectra and Fourier transform of U(VI) doped cementitious materials together with $\mathrm{Ca}$-uranate and uranophane, which are all regarded as reference compounds. The U(VI)-bearing minerals exhibit chemical environments for $\mathrm{U}(\mathrm{VI})$ that could be relevant to $\mathrm{U}(\mathrm{VI})$ doped cementitious systems
Fig. 2 Micro-XRF elemental distribution maps of $\mathrm{U}, \mathrm{Ca}, \mathrm{K}$ and $\mathrm{Fe}$ (top) of a U(VI) doped HCP disk (bottom) prepared from the in-diffusion of U(VI) into the intact sample over 9 months. Selected regions are labeled 1-7 (see text). MicroEXAFS measurements were carried out on spot 1

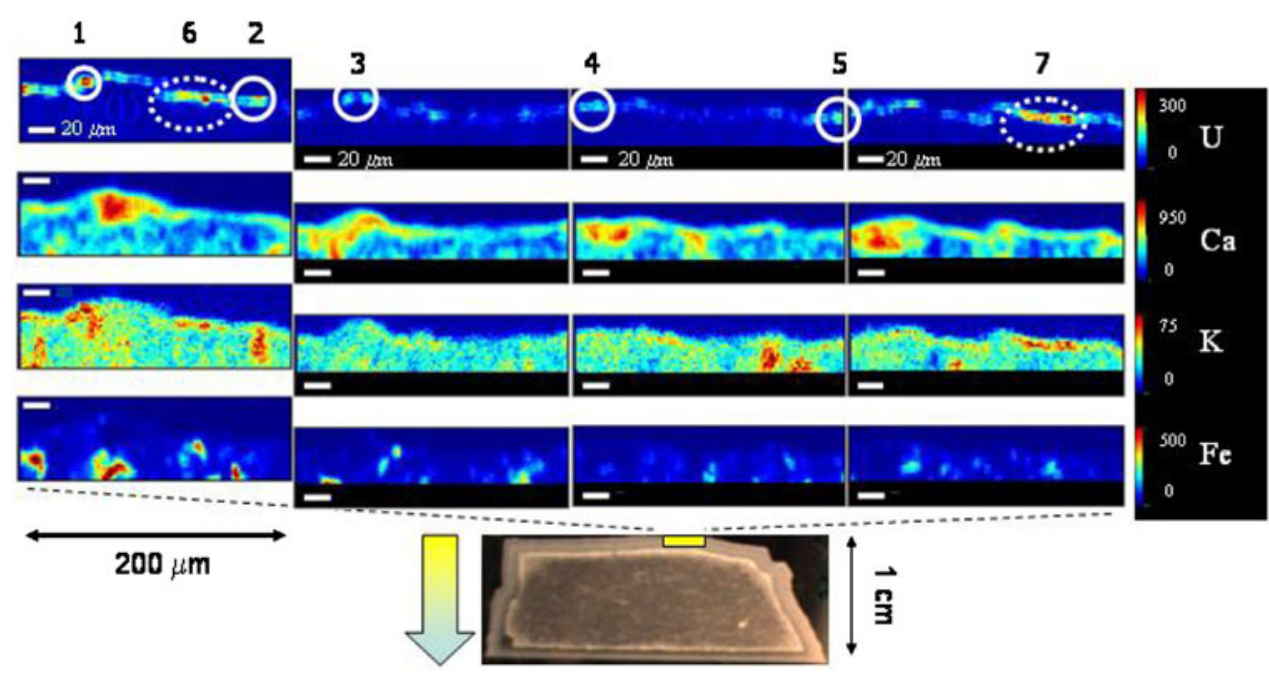


(e.g., [11]). The U(VI)/HCP and U(VI)/C-S-H samples are representative for the chemical environment of $U(V I)$ in sorption samples, i.e., $\mathrm{U}(\mathrm{VI})$ interacting with crushed materials prepared from HCP or synthetic C-S-H, respectively. Short (28 days) and long (309 days) reactions times were chosen, thus covering the time span of the diffusion experiment. Figure 3a reveals that the EXAFS spectra of the U(VI) sorption and diffusion samples are identical. The predominance of only one $\mathrm{U}(\mathrm{VI})$ species in all these samples was further corroborated by principal component analysis using EXAFS data from a larger set of U(VI) doped HCP and C-S-H samples (Macé, Environ. Sci. Technol., submitted). Our observation that the spectra are identical implies that the coordination environment of $\mathrm{U}(\mathrm{VI})$ in all these samples is very similar within about a $5 \AA$ distance around the central uranyl ion probed by EXAFS. This finding shows that both ways of preparing $\mathrm{U}(\mathrm{VI})$ doped cementitious samples, i.e., by sorption and diffusion experiments, gave rise to the same coordination environment of sorbed U(VI) at the atomic level. It should be noted that in the initial stage of the sorption experiments the U(VI) doped HCP and C-S-H suspensions were strongly oversaturated with respect to the solubility- limiting phase, which fixes the U(VI) concentration at $6 \times 10^{-6} \mathrm{M}$. By contrast, the concentration of U(VI) was always fixed at $6 \times 10^{-6} \mathrm{M}$ in the diffusion experiment. Thus, the U(VI) concentration was close to the solubility limit and the system was never oversaturated. Our observation of identical coordination environments of $\mathrm{U}(\mathrm{VI})$ in the sorption and diffusion samples further suggests that $\mathrm{U}(\mathrm{VI})$ diffuses into the HCP material and preferentially interacts with C-S-H phases of the cement matrix.

The structural information summarised in Table 1 was deduced from fitting a merged EXAFS spectra (U_sorbed_ merged) obtained from averaging the spectra of the $\mathrm{U}(\mathrm{VI}) /$ $\mathrm{HCP}$ and $\mathrm{U}(\mathrm{VI}) / \mathrm{C}-\mathrm{S}-\mathrm{H}$ sorption samples (HCP ACW_28d, C-S-H/1.8_H2O_309 d). The merged spectrum was generated because of similar U(VI) structural arrangements in the sorption samples. The structural data show that $\mathrm{U}(\mathrm{VI})$ remains in the form of $\mathrm{UO}_{2}{ }^{2+}$ with two axial oxygen atoms $\left(R_{\mathrm{U}-\mathrm{Oax}}=1.82 \pm 0.02 \AA\right)$ and about four oxygen atoms in the equatorial shell $\left(R_{\mathrm{U}-\mathrm{Oeq}}=2.24 \pm 0.01 \AA\right)$. A neighbouring $\mathrm{Si}$ atom was detected at a distance of about $3.14 \AA$, indicating that $\mathrm{U}(\mathrm{VI})$ was bound to C-S-H at relatively short distance. For the in-diffusion sample only the axial and equatorial oxygen shells could be fitted due to the

Fig. $3 \mathrm{U} \mathrm{L}_{\mathrm{III}}$ edge bulk- and $\mu$-EXAFS measurements on $\mathrm{U}(\mathrm{VI})$ doped HCP and C-S-H samples and reference compounds, a $k^{3}$-weighted experimental (solid line) and theoretical (dotted line) spectra in the $k$-range $2-11 \AA^{-1}, \mathbf{b}$ the corresponding RSFs of experimental (solid line) and fitted (dashed and dotted lines for the imaginary and real part, respectively). Samples: (1) Ca-uranate, (2) U(VI)/HCP sorption sample (HCP_ACW_28d), (3) spot 1 on U(VI) in-diffusion sample (DIFF_ACW_270d), (4) U(VI)/ C-S-H sorption sample (C-S-H/ 1.8_H2O_309d), (5) uranophane


Table 1 Structural information deduced from the merged U(VI) spectra (U_sorbed_merged) obtained by averaging the spectra of the U(VI)/ $\mathrm{HCP}$ and U(VI)/C-S-H sorption samples

\begin{tabular}{llllll}
\hline Sample & Shell & $N$ & $R(\AA)$ & $\sigma^{2}\left(\AA^{2}\right)$ & $\Delta E_{0}(\mathrm{eV})$ \\
\hline U_sorbed_merged & $\mathrm{O}_{\mathrm{ax}}$ & $2.0^{\mathrm{a}}$ & $1.80(2)$ & $0.004(1)$ & $9.6(9)$ \\
& $\mathrm{O}_{\mathrm{eq}}$ & $4.3(5)$ & $2.24(1)$ & $0.005(1)$ & 0.02 \\
& $\mathrm{Si}$ & $0.8(3)$ & $3.14(3)$ & $0.007^{\mathrm{a}}$ &
\end{tabular}

a Fixed parameters; $N$ is the number of atoms; $R$ is the distance between central atom and neighbours $(\AA) ; \sigma$ is the Debye-Waller factor $(\AA) ; \Delta E_{0}$ is the energy shift $(\mathrm{eV}) ; R$-factor is a measure of the fit quality 
low signal-to-noise ratio of the EXAFS spectrum. Coordination numbers and bond distances agreed within the uncertainty with the values listed in Table 1 .

The EXAFS spectra determined for the U(VI) doped cementitious systems further resemble that of U(VI) in the $\mathrm{U}(\mathrm{VI})$ mineral uranophane, suggesting similar coordination environments (Fig. 3a). No phase shift was observed up to a $k$ range of about $9 \AA^{-1}$. This is in contrast to the Cauranate spectrum, which showed significantly different beat patterns in the $k$ range $3.5-4.5 \AA^{-1}$ and at about $6 \AA^{-1}$. In a previous EXAFS study on U(VI) doped C-S-H samples it was postulated that U(VI) bound to C-S-H has a local structure similar to that of $\mathrm{U}(\mathrm{VI})$ in uranophane [11].

\section{Conclusions}

Sorption studies with $\mathrm{U}(\mathrm{VI})$ on $\mathrm{HCP}$ at $\mathrm{pH}=13.3$ show that the uptake kinetics is relatively fast, and equilibrium was reached within a few days. The linear sorption isotherm suggests that only one type of surface site dominates $\mathrm{U}(\mathrm{VI})$ interaction with HCP. Comparison of $R_{d}$ values further suggests that C-S-H phases could be the uptakecontrolling cement phase in HCP. The latter finding is further supported by the spectroscopic investigations on $\mathrm{U}(\mathrm{VI})$ doped HCP and C-S-H samples and, in particular, on an U(VI) in-diffusion sample. Diffusion of U(VI) into a HCP disk causes a heterogeneous distribution of U(VI) in the top surface layer of the disk. U(VI) seems to preferentially accumulate around $\mathrm{Ca}$ rich clinker minerals, presumably belite. Spectroscopic measurements on one U(VI) hot spot show that the coordination environment of U(VI) is similar to that observed for U(VI) sorbed onto HCP and $\mathrm{C}-\mathrm{S}-\mathrm{H}$ phases. Therefore, the $\mathrm{U}(\mathrm{VI})$ speciation is identical regardless of whether $\mathrm{U}(\mathrm{VI})$ uptake starts from oversaturation as in the sorption experiments due to the addition of a highly concentrated U(VI) stock solution or in the diffusion experiments where the U(VI) concentration was fixed at the solubility limit by the U(VI) precipitate formed in the highly alkaline solution.

Acknowledgments The micro-spectroscopic studies were carried out at the Stanford Synchrotron Radiation Lightsource, a national user facility operated by Stanford University on behalf of the US Department of Energy, Office of Basic Energy Sciences. Sam Webb (BL 2-3/SSRL) is thanked for the experimental assistance during the measurements. Thanks are extended to the staff of BM 20 (ROBL) at the European Synchrotron Radiation Facility (ESRF) (Grenoble, France) for assistance with the bulk-EXAFS measurements. Prof. F. P. Glasser is gratefully acknowledged for provision of Ca-uranate and uranophane samples. The study was carried out within the framework of a Marie Curie Fellowship with a grant to N.M. financed by the European Community (EC). Partial financial support was provided by the EC through the 6th framework programme "ACTINET" and by the National Cooperation for the Disposal of Radioactive Waste (Nagra), Switzerland.

\section{References}

1. Chapman N, McCombie C (2003) Principles and standards for the disposal of long-lived radioactive wastes, 1st edn. Elsevier, Oxford

2. Allard B, Eliasson L, Hoglund S, Anderson K (1984) Sorption of Cs, I, and actinides in concrete systems. SKB Technical Report 84-15

3. Bayliss S, McCrohon R, Oliver P, Pilkington NJ, Thomason HP (1996) Near field sorption studies: January 1989 to June 1991, NSS/R227, AEA-ESD-0353

4. Brownsword M, Buchan AB, Ewart FT, McCrohon R, Ormerod GJ, Smith-Briggs JL, Thomason HP (1990) The solubility and sorption of uranium(VI) in a cementitious repository. Mat Res Soc Symp Proc 176:577-582

5. Wieland E, Van Loon LR (2002) Cementitious near-field sorption database for performance assessment of an ILW repository in Opalinus clay. Nagra Technical Report NTB 02-20, Wettingen

6. Sutton M, Warwick P, Hall A (2003) Uranium(VI) interactions with OPC/PFA grout. J Environ Monit 5:922-925

7. Pointeau I, Landesman C, Giffault E, Reiller P (2004) Reproducibility of the uptake of $\mathrm{U}(\mathrm{VI})$ onto degraded cement pastes and calcium silicate hydrates. Radiochim Acta 92:645-650

8. Tits J, Fujita T, Tsukamoto M, Wieland E (2008) Uranium(VI) uptake by synthetic calcium silicate hydrates. Mat Res Soc Symp Proc 1107:467-474

9. Sylvester ER, Allen PG, Zhao P, Viani BE (2000) Interactions of uranium and neptunium with cementitious materials studied by XAFS. Mat Res Soc Symp Proc 608:307-312

10. Zhao P, Allen PG, Sylvester ER, Viani BE (2000) The partitioning of uranium and neptunium onto hydrothermally altered concrete. Radiochim Acta 66:729-736

11. Harfouche M, Wieland E, Dähn R, Fujita T, Tits J, Kunz D, Tsukamoto M (2006) EXAFS study of U(VI) uptake by calcium silicate hydrates. J Colloid Interface Sci 303:195-204

12. Sarott F-A, Bradbury MH, Pandolfo P, Spieler P (1992) Diffusion and adsorption studies on hardened cement paste and the effect of carbonation on diffusion rates. Cem Concr Res 22:439-444

13. Tits J, Jakob A, Wieland E, Spieler P (2003) Diffusion of tritiated water and ${ }^{22} \mathrm{Na}^{+}$through non-degraded hardened cement paste. J Contam Hydrol 61:45-62

14. Lothenbach B, Wieland E (2006) A thermodynamic approach to the hydration of sulphate-resisting Portland cement. Waste Manag 26:706-719

15. Tits J, Wieland E, Müller CJ, Landesman D, Bradbury MH (2006) Strontium binding by calcium silicate hydrates. J Colloid Interface Sci 300:78-87

16. Wieland E, Tits J, Ulrich A, Bradbury MH (2006) Experimental evidence for the solubility limitation of the aqueous $\mathrm{Ni}(\mathrm{II})$ concentration and isotopic exchange of ${ }^{63} \mathrm{Ni}$ in cementitious systems. Radiochim Acta 94:29-36

17. Wieland E, Tits J, Kunz D, Dähn R (2008) Strontium uptake by cementitious materials. Environ Sci Technol 42:403-409

18. Jakob A. (2002) Diffusion of tritiated water (HTO) and ${ }^{22} \mathrm{Na}^{+}-$ ions through non-degraded hardened cement pastes-II. Modelling results, PSI Bericht Nr. 02-21, Villigen PSI

19. Moroni LP, Glasser FP (1995) Reactions between cement components and U(VI) oxide. Waste Manag 15:243-354

20. Webb SM (2005) Sixpack: a graphical user interface for XAS analysis using IFEFFIT. Phys Scr T115:1011-1014

21. Ravel B, Newville M (2005) ATHENA, ARTEMIS, HEPHA ESTUS: data analysis for X-ray absorption spectroscopy using IFEFFIT. J Synchrotron Rad 12:537-541

22. Newville M (2001) EXAFS analysis using FEFF and FEFFIT. J Synchrotron Rad 8:96-100 
23. Hudson EA, Allen PG, Terminello LJ, Denecke MA, Reich T (1996) Polarized x-ray-absorption spectroscopy of the uranyl ion: comparison of experiment and theory. Phys Rev B 54:156-165

24. Ochs M, Talerico C, Lothenbach B, Giffault E (2003) Systematic trends and empirical modelling of lead uptake by cements and cement minerals. Mat Res Soc Symp Proc 757:693-698

25. Bayliss S, Ewart FT, Howse RM, Smith-Briggs JL, Thomason HP, Willmott HA (1988) The solubility and sorption of lead-210 and carbon-14 in a near field environment. Mat Res Soc Symp Proc 112:33-42

26. Evans N (2008) Binding mechanisms of radionuclides to cement. Cem Concr Res 38:543-553
27. Glasser FP (1993) In: Spence RD (ed) Chemistry and microstructure of solidified waste forms. Lewis Publishers, Boca Raton 28. Vespa M, Dähn R, Gallucci E, Grolimund D, Wieland E, Scheidegger AM (2006) Microscale investigations of Ni uptake by cement using a combination of scanning electron microscopy and synchrotron-based techniques. Environ Sci Technol 40:7702-7709

29. Mandaliev P, Dähn R, Wehrli B, Wieland E (2009) Macro- and microspectroscopic study of $\mathrm{Nd}(\mathrm{III})$ uptake mechanisms by hardened cement paste. Environ Sci Technol 43:8462-8468 